\title{
Central cholinergic activation of a vagus nerve-to-spleen circuit alleviates experimental colitis
}

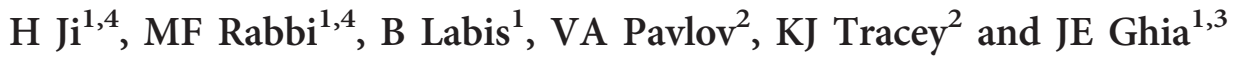

The cholinergic anti-inflammatory pathway is an efferent vagus nerve-based mechanism that regulates immune responses and cytokine production through $\alpha 7$ nicotinic acetylcholine receptor ( $\alpha 7 \mathrm{nAChR})$ signaling. Decreased efferent vagus nerve activity is observed in inflammatory bowel disease. We determined whether central activation of this pathway alters inflammation in mice with colitis and the mediating role of a vagus nerve-to-spleen circuit and $\alpha 7 n A C h R$ signaling. Two experimental models of colitis were used in C57BL/6 mice. Central cholinergic activation induced by the acetylcholinesterase inhibitor galantamine or a muscarinic acetylcholine receptor agonist treatments resulted in reduced mucosal inflammation associated with decreased major histocompatibility complex II level and pro-inflammatory cytokine secretion by splenic $\mathrm{CD}_{11 \mathrm{c}}{ }^{+}$cells mediated by $\alpha 7 \mathrm{nAChR}$ signaling. The cholinergic anti-inflammatory efficacy was abolished in mice with vagotomy, splenic neurectomy, or splenectomy. In conclusion, central cholinergic activation of a vagus nerve-to-spleen circuit controls intestinal inflammation and this regulation can be explored to develop novel therapeutic strategies.

\section{INTRODUCTION}

Inflammatory bowel diseases (IBDs), consisting of Crohn's disease (CD) and ulcerative colitis (UC), are characterized by a chronic relapsing and remitting course as a result of intestinal inflammation. ${ }^{1}$ The release of inflammatory mediators, including pro-inflammatory cytokines, from immune cells mediate tissue injury and exacerbation of IBD. ${ }^{2}$ Accordingly, several therapeutic approaches targeting inflammatory cytokines in IBDs have been investigated. ${ }^{3}$ Cholinergic signaling along the vagus nerve has been shown to control interleukin (IL)-6, IL$1 \beta$, tumor necrosis factor (TNF)- $\alpha$, and other pro-inflammatory cytokine production in different inflammatory conditions, including IBD. ${ }^{4-6} \alpha 7$ Nicotinic acetylcholine receptors $(\alpha 7 \mathrm{nAChR})$ on macrophages, monocytes, and mast cells have been shown to mediate cholinergic anti-inflammatory output. ${ }^{7,8}$ This regulation is a part of the current working model of the "inflammatory reflex" controlling immune responses and cytokine levels. ${ }^{9}$ In this model, afferent vagus neurons sensing peripheral inflammatory molecules convey the signal to the brain. ${ }^{9}$ Consequent activation of efferent vagus neurons results in increased cholinergic $\alpha 7 \mathrm{nAChR}$-dependent anti-inflammatory output and suppressed pro-inflammatory cytokine release. Recent findings have suggested a role for the splenic nerve and the spleen in this cholinergic anti-inflammatory pathway. ${ }^{8}$

IBD is associated with an autonomic imbalance and up to $35 \%$ of patients with UC exhibit autonomic dysfunction with impaired efferent vagus nerve activity. ${ }^{10}$ Although current animal models do not sufficiently recapitulate IBD, we have previously reported that the vagus nerve has a tonic inhibitory role on acute inflammation in murine models of colitis mimicking UC (dextran sulfate sodium (DSS) model) and CD (2, 4 dinitrobenzenesulfonic acid (DNBS) model). ${ }^{4}$ In this context, the absence of the vagus nerve worsened acute DSS and DNBS colitis through a macrophage-mediated mechanism, associated with the release of higher levels of IL-6, IL-1 $\beta$, and TNF- $\alpha$, without affecting the level of the anti-inflammatory cytokine IL-10. Electrical vagus nerve stimulation suppresses myeloperoxidase activity (a marker of neutrophil infiltration) and TNF- $\alpha$ inflammatory cytokine levels in experimental colitis and endotoxemia, respectively. ${ }^{11}$ Moreover, in line with

\footnotetext{
${ }^{1}$ Department of Immunology and Internal Medicine Section of Gastroenterology, University of Manitoba, Winnipeg, Manitoba, Canada. ${ }^{2}$ Center for Biomedical Science, The Feinstein Institute for Medical Research, Manhasset, New York, USA and ${ }^{3}$ Department of Medicine, McMaster University, Hamilton, Ontario, Canada. Correspondence: JE Ghia (jeghia@yahoo.fr)

${ }^{4}$ These authors, listed in alphabetical order, contributed equally to this work.
}

Received 29 January 2013; accepted 20 June 2013; published online 24 July 2013. doi:10.1038/mi.2013.52 
the role of $\alpha 7 \mathrm{nAChR}$ in mediating anti-inflammatory cholinergic signals, it has been demonstrated that smoking ameliorates inflammation in UC patients. ${ }^{12}$ Conversely, smoking exacerbates inflammation in $\mathrm{CD} .{ }^{12,13}$

Dendritic cells (DCs) are key cells of the innate immune system that bridge innate with adaptive immune responses. Strategically positioned in the lamina propria in proximity to a number of luminal bacteria and antigenic stimuli, these cells perform a key role in activation of the immune response, generation of gut inflammation via their passage into the spleen, and interaction with T cells. ${ }^{14}$ Human studies have revealed that there is a significant increase in the numbers of antigen-presenting cell, including DCs within the inflamed tissue and the peripheral blood of patients with CD or UC. ${ }^{15,16}$ Furthermore, DCs depletion in DSS-treated CD11c-DT receptor transgenic mice almost completely inhibited experimental colitis. ${ }^{17}$ Nicotinic receptors, including $\alpha 7 \mathrm{nAChR}$, are also expressed by human monocytes ${ }^{18}$ and mouse DCs. ${ }^{19}$

The cholinergic anti-inflammatory pathway can be activated in the central nervous system by muscarinic acetylcholine receptor $(\mathrm{mAChR})$ ligands or acetylcholinesterase (AChE) inhibitors. ${ }^{20,21}$ Galantamine (GAL) is a reversible, competitive AChE inhibitor, which crosses the blood-brain barrier, increases brain cholinergic network activity ${ }^{22}$ and is widely used in the treatment of Alzheimer's disease. GAL activates efferent vagus nerve activity $^{23}$ and its anti-inflammatory activity has been associated with brain $\mathrm{mAChR}$-mediated activation of the cholinergic anti-inflammatory pathway. ${ }^{21}$ In addition, it was very recently demonstrated that a treatment with another centrally acting cholinesterase inhibitorrivastigmine-suppresses IL-6 levels and decreases the severity of murine DSS- and trinitrobenzene sulfonic acid (TNBS)induced colitis. ${ }^{24} \mathrm{~A}$ role for $\mathrm{mAChRs}$ in the central nervous system and macrophages in mediating the effects of rivastigmine was also indicated.

Recent findings have highlighted a key role of the spleen in mediating vagus nerve anti-inflammatory signaling during endotoxemia. ${ }^{25}$ In the context of endotoxemia, the absence of the intact vagus nerve or the spleen results in abrogation of the beneficial effect of the vagus nerve activation. However, the implication of a vagus nerve-to-spleen anti-inflammatory axis in the regulation of intestinal inflammation remains to be determined. To provide insight, here we studied whether central activation of the cholinergic anti-inflammatory pathway by the AChE inhibitor GAL or $\mathrm{MAChR}$ ligands alters the severity of DSS colitis and the specific role of the spleen and DCs. We report that treatments with GAL or McN-A-343 (a M1mAChR agonist) significantly ameliorate disease severity and inhibit inflammation in the context of experimental colitis. These effects were entirely dependent on vagus nerve and splenic nerve integrity and associated with inhibition of splenic $\mathrm{CD}_{11 \mathrm{c}^{+}}$cell pro-inflammatory cytokine production. In line with the importance of $\alpha 7 \mathrm{nAChR}$-mediated signaling in the cholinergic regulation of inflammation, we found that direct stimulation of DCs with a $\alpha 7 \mathrm{nAChR}$ agonist decreases the release of pro-inflammatory cytokines. Similar cholinergic anti-inflammatory mechanisms were demonstrated using the DNBS model, indicating the broader scope of our findings in the regulation of intestinal inflammation.

\section{RESULTS}

\section{Centrally acting AChE inhibitor treatment ameliorates the severity of colitis}

Previous findings have characterized GAL as a central activator of the cholinergic anti-inflammatory pathway. ${ }^{21,23,26}$ Daily administration of GAL (1-4 mg kg ${ }^{-1}$ day $^{-1}$, intraperitoneal (IP)), starting 1 day before disease induction, dose-dependently reduced the severity of colitis (Figure 1a-d). GAL-treated mice with colitis showed a significantly lower disease activity index for the last 2 days as compared with saline-treated controls with the disease (Figure 1a). This drug effect was dose-dependent, and the highest reduction was achieved with a dose of $4 \mathrm{mg} \mathrm{kg}^{-1} \mathrm{day}^{-1}$, which has been previously shown to inhibit mouse brain AChE activity by $43 \%{ }^{26}$ The decreased severity of colitis in GAL $\left(4 \mathrm{mg} \mathrm{kg}^{-1}\right.$ day $\left.{ }^{-1}\right)$-treated mice as compared with saline-treated controls was also further demonstrated by the 3.2 -fold decrease in the macroscopic damage score, in the 3.4-fold decrease in myeloperoxidase (MPO) activity, and in the 1.25-fold decrease in serum C-reactive protein levels (Figure 1b-d). GAL $\left(4 \mathrm{mg} \mathrm{kg}^{-1}\right.$ day $^{-1}$ ) treatment of mice with DSS-induced colitis also significantly lowered the histological manifestation of the disease (Figure $\mathbf{2 b}, \mathbf{c}, \mathbf{g}$ ). Colonic IL-1 $\beta$ levels in GAL-treated mice were fourfold lower as compared with the DSS-saline treated group (Figure 3d). Accordingly, colonic IL-6 and TNF$\alpha$ levels were 2- and 2.3-fold lower (Figure 3e,f). No significant changes were detected for colonic IL-10 (see Supplementary Figure S1A online).

To characterize the specificity of brain AChE inhibition in preventing the development of colitis, we utilized Huperzine-A (Hup A), a structurally distinct, highly selective, centrally acting AChE inhibitor. Hup A administration $\left(0.4 \mathrm{mg} \mathrm{kg}^{-1}\right.$ day $^{-1}$, IP) significantly reduced the disease severity and inflammation in mice with DSS colitis (see Supplementary Table S1 online; Figure 2d,g). In addition to their efficacy in DSS colitis, GAL and Hup A treatments decreased the severity of DNBS colitis (see Supplementary Figure S2 online). Taken together, these findings indicate that centrally acting AChE inhibitor treatments result in disease-alleviating and counter-inflammatory effects in two experimental models of colitis.

\section{Central muscarinic receptors are essential for alleviation of colitis and inhibition of colonic inflammation by an AChE inhibitor}

We next studied whether central mAChRs, which critically mediate brain cholinergic pathways and vagus nerve activation, ${ }^{21}$ are required for the anti-inflammatory effect of GAL. Pretreatment with atropine methyl nitrate (AMN), an mAChR antagonist that does not cross the blood-brain barrier, failed to alter the beneficial effects of GAL on colitis (Figure 3). In contrast, pretreatment with atropine sulfate (AS), an mAChR antagonist that penetrates the blood-brain barrier, abrogated 
a

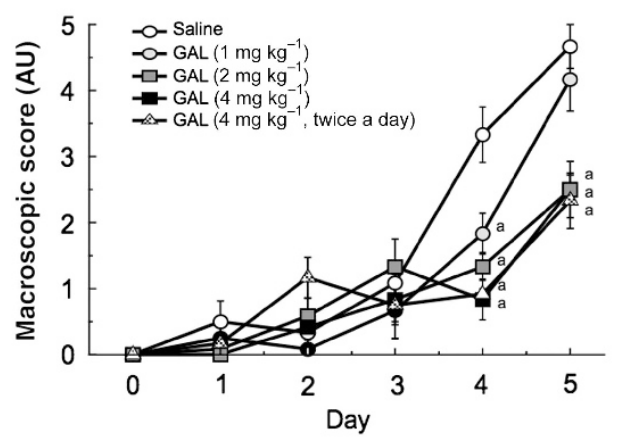

c

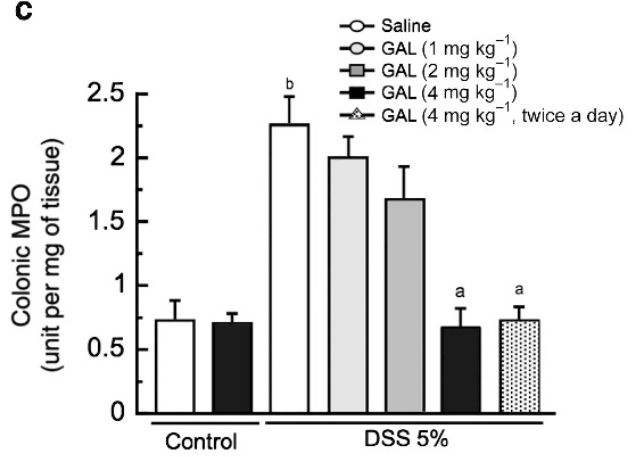

b

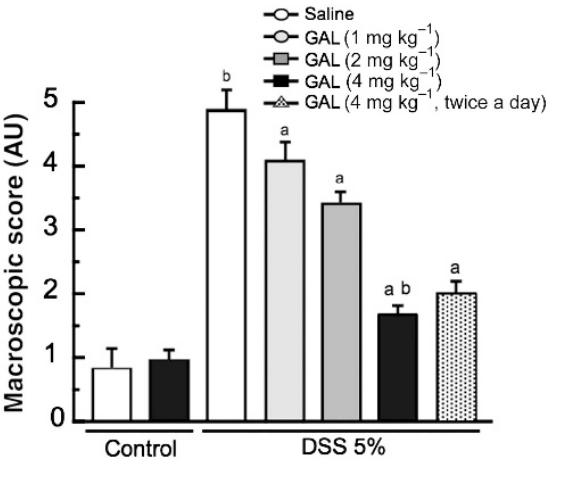

d

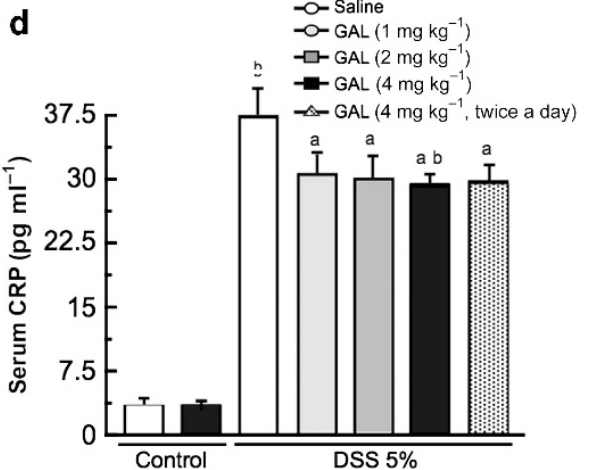

Figure 1 Galantamine (GAL) alleviates the severity of dextran sulfate sodium (DSS)-induced colitis. GAL (6 days, intraperitoneal (IP)) treatment was started 1 day before colitis induction. (a) Disease activity index; (b) macroscopic scores; (c) colonic myeloperoxidase (MPO) activity;

(d) serum C-reactive protein (CRP). Values are shown as means \pm s.e.m. Samples were collected on day 5 post DSS; mice per group $\geqslant 8$. ${ }^{\text {a }} P<0.05$ as compared with the saline DSS-treated group, ${ }^{b} \mathrm{P}<0.05$ as compared with the control $\left(\mathrm{H}_{2} \mathrm{O}\right)$-treated group. AU, arbitrary units.

the suppressive GAL effect on colitis development (Figure 3). No significant differences were detected in colonic IL-10 levels (see Supplementary Figure S1A online). Neither AMN nor AS alone significantly modified the expression of colitis. These results indicate that the anti-inflammatory effects of GAL in the context of colitis are mediated through a brain mAChR-dependent mechanism. Although the presence of mAChRs has been reported on immune cells, ${ }^{19}$ our data indicate that direct modulation of peripheral mAChRs has no significant effect on the magnitude of colonic inflammation. In the context of DNBS colitis, results were similar for MPO activity (see Supplementary Figure S2A online). However, AS and AMN treatments resulted in a slight but statistically significant decrease in colonic IL-1 $\beta$ levels (see Supplementary Figure S2B online).

\section{Central administration of mAChR ligands attenuates the severity of colitis and inhibits colonic inflammation}

As central mAChRs are important in mediating the effects of GAL, we further assessed the regulatory involvement of these receptors in the context of colitis. Previously, a role for M1mAChR activation and M2mAChR inhibition in the regulation of inflammation during endotoxemia has been described. ${ }^{20}$ Accordingly, we performed experiments with mice subjected to intracerebroventricular (ICV) infusions of specific mAChR ligands. Treatment with the M1mAChR agonist (McN-A-343; $5 \mathrm{ng} \mathrm{kg}^{-1}$ day $^{-1}$ ) starting 1 day before the induction of DSS colitis decreased the macroscopic score, the
MPO activity, the serum C-reactive protein levels, the colonic tissue pro-inflammatory cytokine levels (Figure 4), and the histological score (Figure 2e,g). No significant differences were detected in the colonic IL-10 levels (see Supplementary Figure S1B online).

Then, we examined the anti-inflammatory efficacy of an alternative approach of central cholinergic activation. Central $\mathrm{ACh}$ release is negatively regulated by the presynaptic M2mAChR autoreceptor. ${ }^{27,28}$ Therefore, we studied the efficacy of ICV infusion of the M2mAChR antagonist methoctramine (MTT; $5 \mathrm{ng} \mathrm{kg}^{-1}$ day $^{-1}$ ). MTT administration, initiated 1 day before induction of colitis resulted in comparable significant decrease of colitis severity (Figures 4 and $\mathbf{2} \mathbf{f}, \mathbf{g}$ ). We also demonstrated the beneficial effect of both treatments in the context of DNBS colitis (see Supplementary Figure S3 online). These findings highlight the specific regulatory role of central $\mathrm{mAChRs}$ in suppressing colitis pathogenesis and are in line with the previously reported antiinflammatory effects of these two drugs in the context of endotoxemia. $^{20}$

\section{Central cholinergic disease-alleviating and anti- inflammatory efficacy is mediated through vagus and} splenic nerve signaling and the release of $\mathrm{ACh}$ in the spleen To study whether the beneficial effects of central cholinergic activation in the context of colitis depend on the vagus nerveto-spleen axis, we performed a series of experiments with mice 


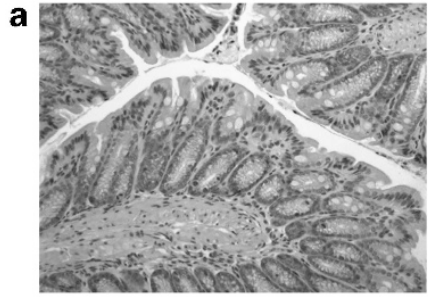

Control

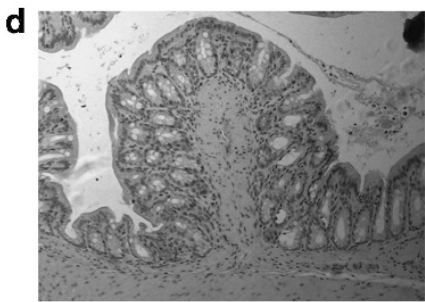

GAL 2 mg kg-1 + DSS 5\%

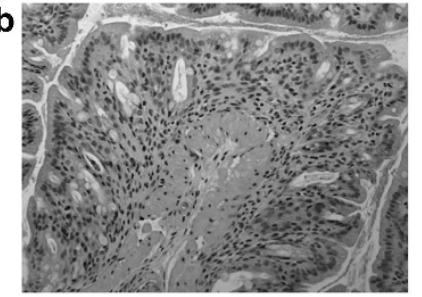

DSS $5 \%$

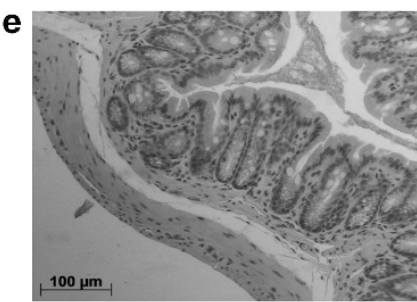

GAL 4 mg kg-1 + DSS 5\%

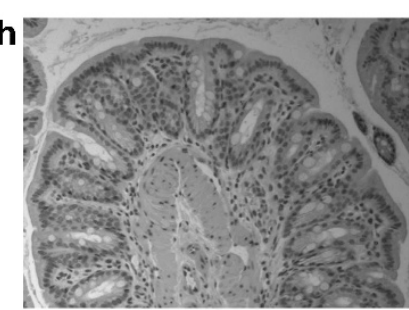

McN-A-343 $5 \mathrm{ng} \mathrm{kg}^{-1}+$ DSS 5\%

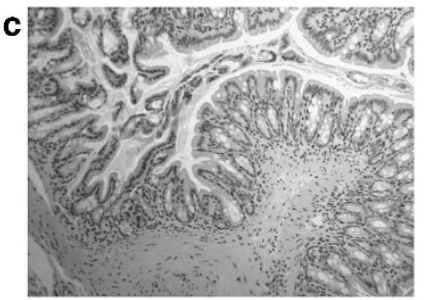

GAL $1 \mathrm{mg} \mathrm{kg}^{-1}+$ DSS 5\%

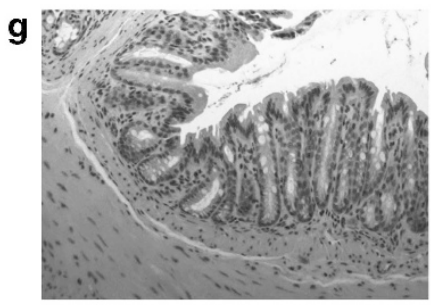

Hup A $0.4 \mathrm{mg} \mathrm{kg}^{-1}+$ DSS 5\%

j
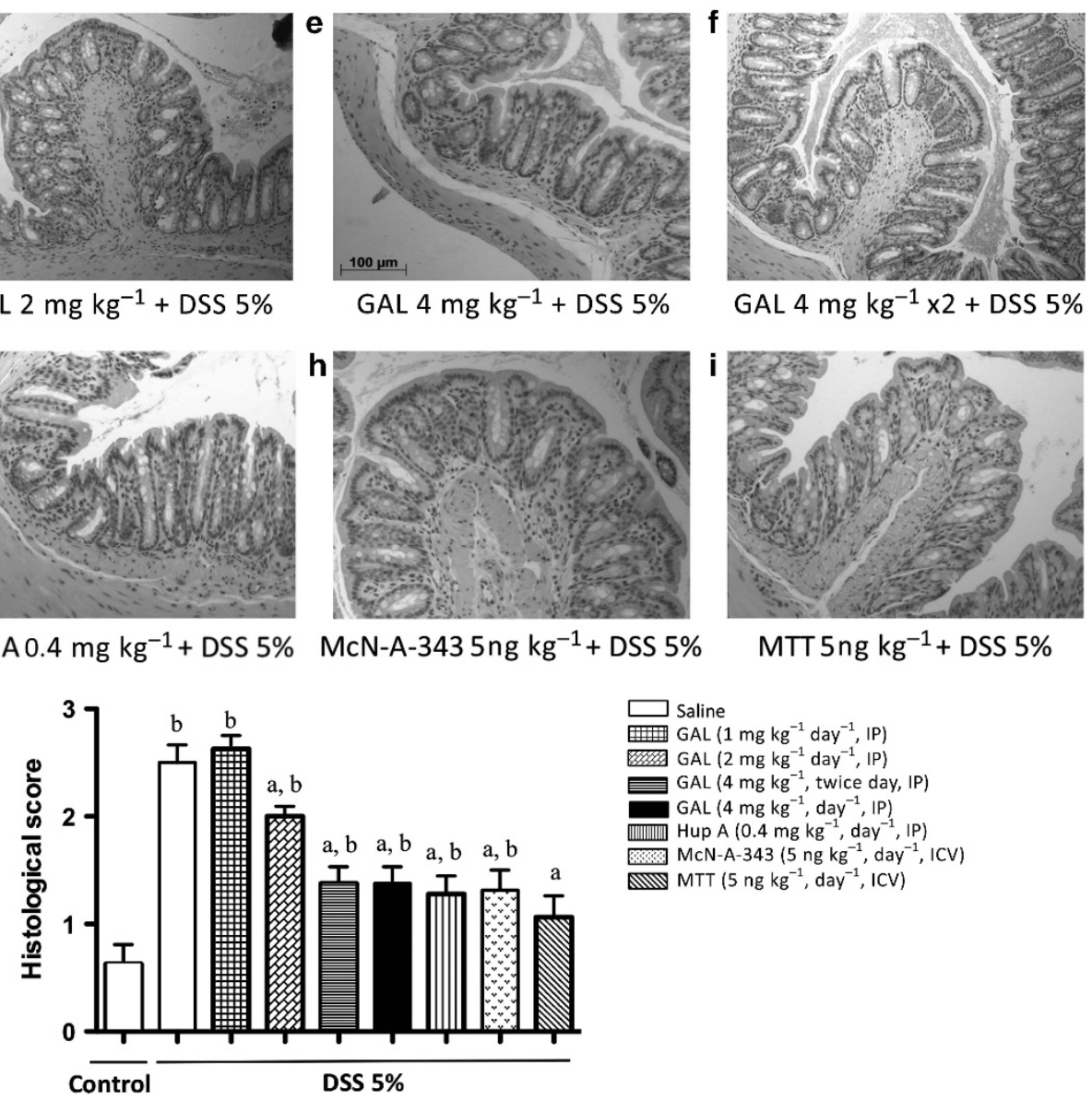

GAL $4 \mathrm{mg} \mathrm{kg}^{-1} \times 2+$ DSS $5 \%$

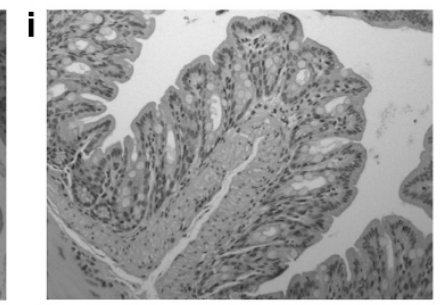

MTT 5ng kg-1 + DSS 5\%

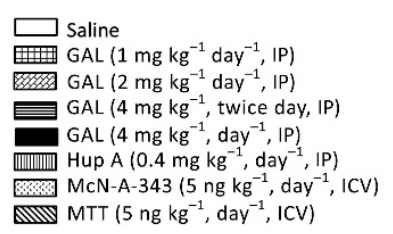

Figure 2 Centrally acting acetylcholinesterase inhibitors and mAChR ligands alleviate the severity of dextran sulfate sodium (DSS)-induced colitis. Appearance of a colon in mice (a) in the absence of colitis (control group); (b) in mice with DSS-induced colitis; (c) in galantamine (GAL) $\left(4 \mathrm{mg} \mathrm{kg}^{-1}\right.$, intraperitoneal (IP) for 6 days)-treated mice with DSS-induced colitis; (d) in Huperzine A (Hup A) $\left(0.4 \mathrm{mg} \mathrm{kg}^{-1}\right.$, IP for 6 days)-treated mice with DSS-induced colitis; (e) in McN-A-343 (M1mAChR agonist) $\left(5 \mathrm{ng} \mathrm{kg}^{-1}\right.$ day $^{-1}$, intracerebroventricular (ICV), for 6 days)-treated mice with DSS-induced colitis; (f) in methoctramine (MTT, M2mAChR antagonist) $\left(5 \mathrm{ng} \mathrm{kg}^{-1}\right.$ day $^{-1}$, ICV, for 6 days)-treated mice with DSS-induced colitis; (g) histological score; and values are shown as means \pm s.e.m. Samples were collected on day 5 post-DSS treatment; mice per group $\geqslant 8$. ${ }^{\mathrm{a}} \mathrm{P}<0.05$ as compared with the saline DSS-treated group, ${ }^{\mathrm{b}} \mathrm{P}<0.05$ as compared with the control $\left(\mathrm{H}_{2} \mathrm{O}\right)$-group. Hematoxylin and eosin staining, $\times 100$ magnification.

subjected to vagotomy (VXP), splenic neurectomy (NRX), and/ or splenectomy (SPX). VXP significantly exacerbated the severity of colitis (Figure 5 and see Supplementary Figure S4 online), thus confirming our previously reported findings. ${ }^{29}$ SPX did not alter the development of colitis; however, the absence of the spleen abolished the deleterious effect of VXP (Figure 5 and see Supplementary Figure S4 online). The beneficial effects of GAL on disease severity and inflammation were abolished in mice with VXP, SPX, or VXP and SPX (Figure 5 and see Supplementary Figure S4 online). NRX resulted in exacerbation of disease severity comparable to VXP
(Figure 5 and see Supplementary Figure S4 online). These effects were abolished in mice simultaneously subjected to SPX (Figure 5 and see Supplementary Figure S4 online). Furthermore, the beneficial effects of GAL were abrogated in mice with NRX, SPX, or NRX and SPX (Figure 5; seeSupplementary Figure S4 online). No effect of these manipulations was observed on colonic IL-10 levels (see Supplementary Figure S1 online).

In addition, beneficial effects of the M1mAChR agonist McN-A-343 ICV infusion in the context of colitis were abolished in the absence of an intact vagus nerve or splenic 

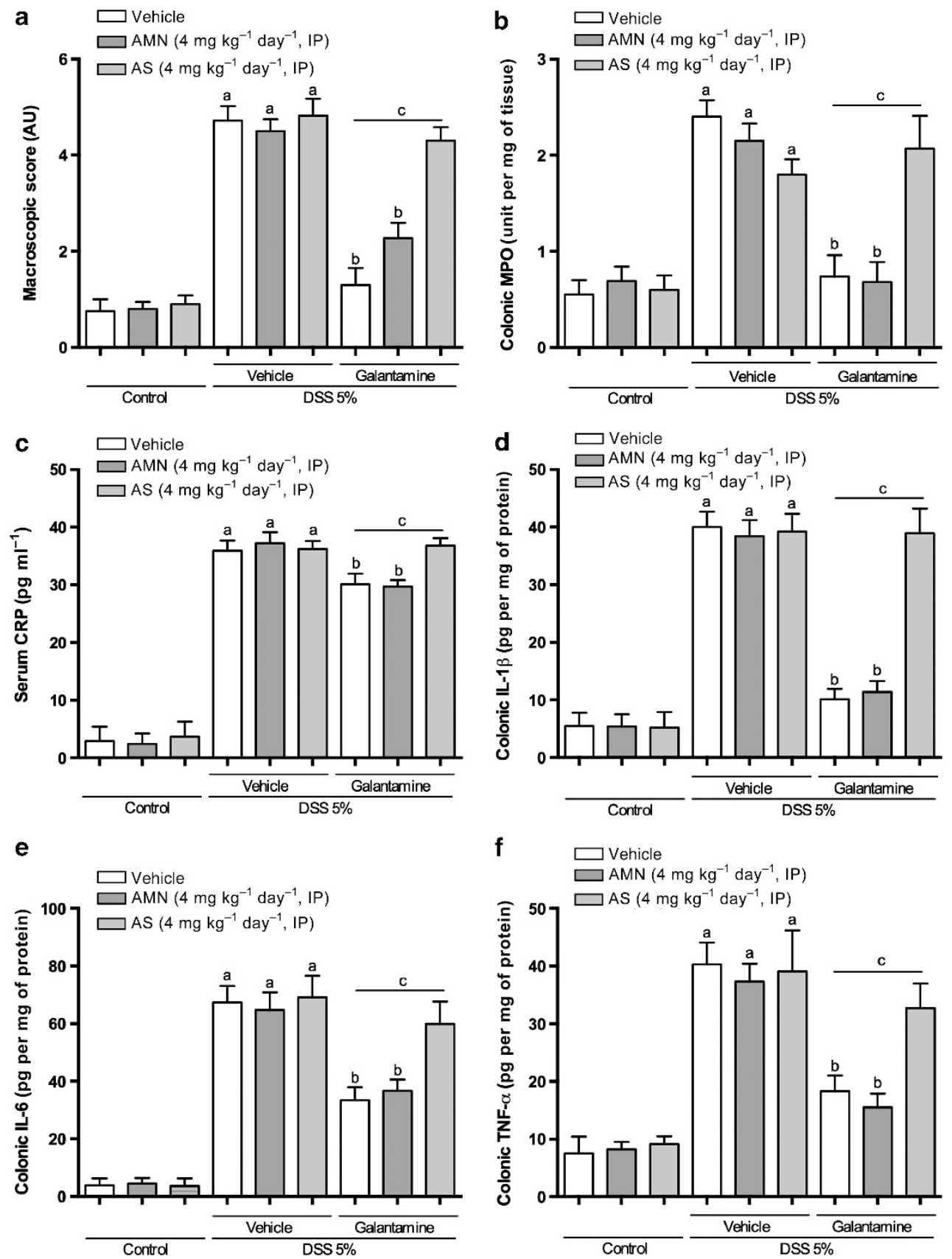

Figure 3 Galantamine (GAL) effects in dextran sulfate sodium (DSS)-induced colitis are mediated through central mAChRs. Atropine methyl nitrate (AMN, a mAChR antagonist that does not cross the blood-brain barrier) ( $4 \mathrm{mg} \mathrm{kg}^{-1}$, intraperitoneal (IP) for 6 days) or atropine sulfate (AS, a mAChR antagonist that crosses the blood-brain barrier) were administered as a single daily injection 20 min before every GAL administration (4 mg kg ${ }^{-1}$, IP, for 6 days). (a) Macroscopic score; (b) colonic myeloperoxidase (MPO) activity; (c) serum C-reactive protein (CRP); (d) colonic interleukin (IL)-1 $\beta$ amount; (e) colonic IL-6 amount; and (f) colonic tumor necrosis factor (TNF)- $\alpha$ amount. Values are shown as means \pm s.e.m. Samples were collected on day 5 post-DSS treatment; mice per group $\geqslant 8$. ${ }^{a} P<0.05$, compared with the non-DSS-treated group (control, $\mathrm{H}_{2} \mathrm{O}$ ), ${ }^{\mathrm{b}} P<0.05$ as compared with the vehicle DSS-treated group, ${ }^{\mathrm{C}} P<0.05$ as compared with the GAL-DSS-treated group. AU, arbitrary units.

nerve or in mice with SPX (see Supplementary Figure S5 online). Previously, vagus nerve activation functionally associated with signaling along the splenic nerve has been demonstrated to result in an increase of ACh in the spleen. ${ }^{25}$ Therefore, we next examined the effects of GAL and McN-A343 treatments on splenic ACh levels and the mediating role of the vagus nerve and the splenic nerve. GAL and McN-A-343 treatments were associated with a significant increase in ACh levels in the spleen of sham-operated control mice with DSS colitis (Figure 6). ACh levels in the VXP and the NRX group were significantly lower as compared with sham-operated controls in mice with DSS colitis, and VXP and NRX significantly abolished the increase in splenic ACh release caused by GAL or McN-A-343 treatments (Figure 6). 

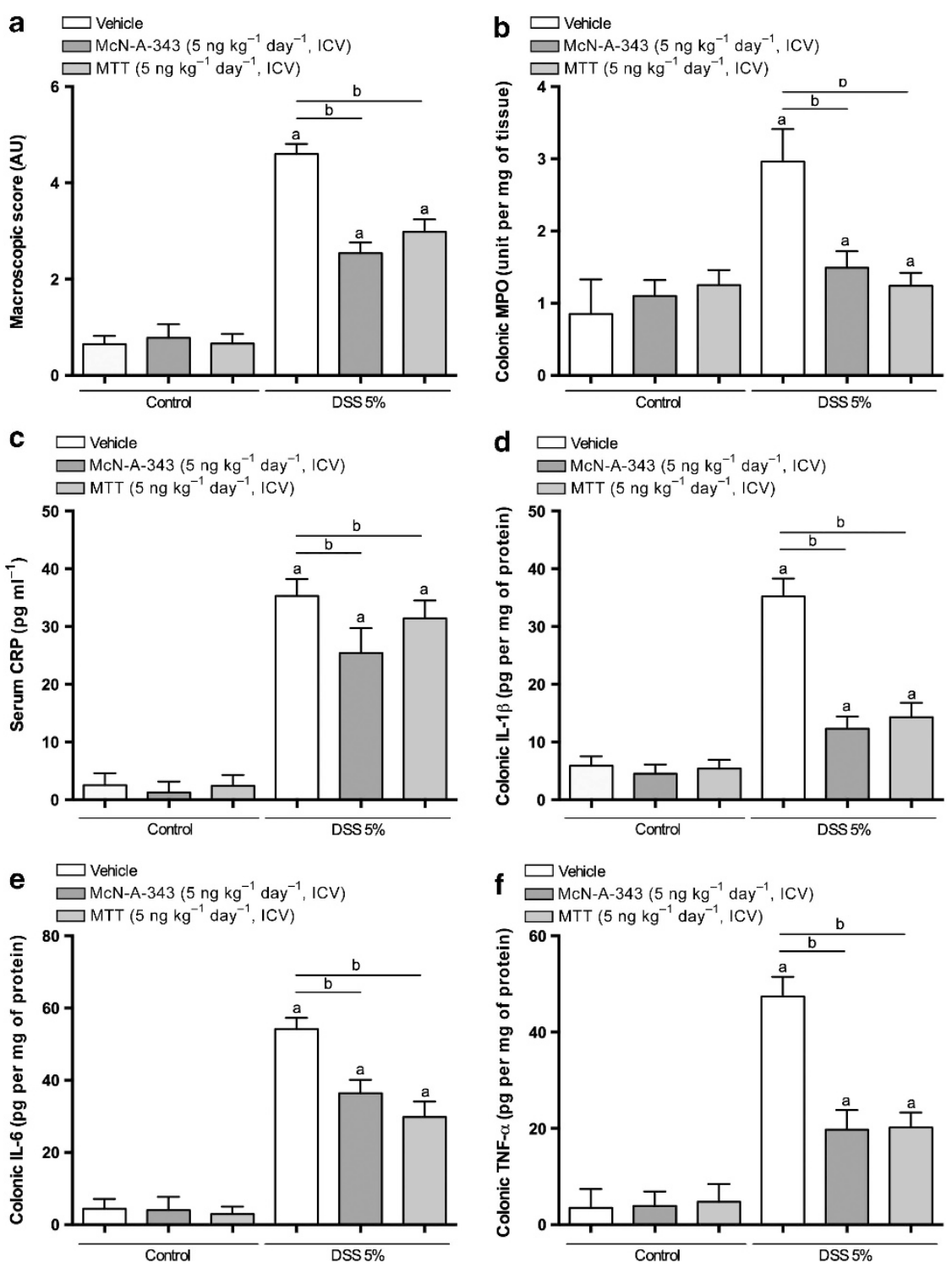

Figure 4 Central administration of a M1mAChR agonist or a M2mAChR antagonist alleviates the severity of dextran sulfate sodium (DSS)-induced colitis. McN-A-343 (M1mAChR agonist) or methoctramine (MTT, M2mAChR antagonist) $\left(5 \mathrm{ng} \mathrm{kg}^{-1}\right.$ day $^{-1}$, intracerebroventricular (ICV), for 6 days) treatments were started 1 day before induction of colitis. (a) Macroscopic score; (b) colonic myeloperoxidase (MPO) activity; (c) serum C-reactive protein (CRP); (d) colonic interleukin (IL)-1 $\beta$ amount; (e) colonic IL-6 amount; and (f) colonic tumor necrosis factor (TNF)- $\alpha$ amount. Values are shown as means \pm s.e.m. Samples were collected on day 5 post-DSS treatment; mice per group $\geqslant 8$. ${ }^{a} P<0.05$, compared with the non-DSS-treated group (control, $\mathrm{H}_{2} \mathrm{O}$ ), ${ }^{\mathrm{b}} \mathrm{P}<0.05$ as compared with the vehicle DSS-treated group. $\mathrm{AU}$, arbitrary units.

Together, these results demonstrate that neural signaling through the vagus nerve-to-spleen axis is required for central cholinergic activation to alleviate colitis.

\section{Central cholinergic activation decreases splenic CD11c ${ }^{+}$ cell cytokine production}

To gain further insight into the cellular mechanisms mediating cholinergic anti-inflammatory effects on colitis, we studied the role of splenic CD11 $\mathrm{c}^{+}$cells. IL-12p40, IL-1 $\beta$, and IL-6 levels in the splenic $\mathrm{CD} 11 \mathrm{c}^{+}$cells' culture supernatant from the colitic GAL-treated groups (Figure 7) were significantly decreased as compared with the colitic non-treated groups. Conversely, VXP or NRX increased IL-12p40, IL-1 $\beta$, and IL-6 levels (Figure 7) and no beneficial effect of GAL (Figure 7) treatment was found in the absence of an intact vagus or splenic nerve. The cell viability in non-colitic condition was $99+2.4 \%$; however, in all the groups treated with DSS, the cell viability decreased to $82+3.2 \%$. We next performed ex vivo experiments to highlight the role of the $\alpha 7 \mathrm{nAChR}$ in mediating cholinergic antiinflammatory effects in the spleen. Splenic CD11c ${ }^{+}$cells were isolated from colitic mice subjected to sham operation, VXP or NRX, and a treatment with GAL (IP). Then, these cells were treated ex vivo with GTS-21 (a specific $\alpha 7 \mathrm{nAChR}$ agonist). At all conditions, the addition of GTS-21 in the culture medium significantly decreased the levels of IL-12p 40 , IL-1 $\beta$, and IL-6 production (Figure 7). We also demonstrated the beneficial effects of cholinergic treatments in the context of DNBS colitis (see Supplementary Figure S6 online). Apart from being an 

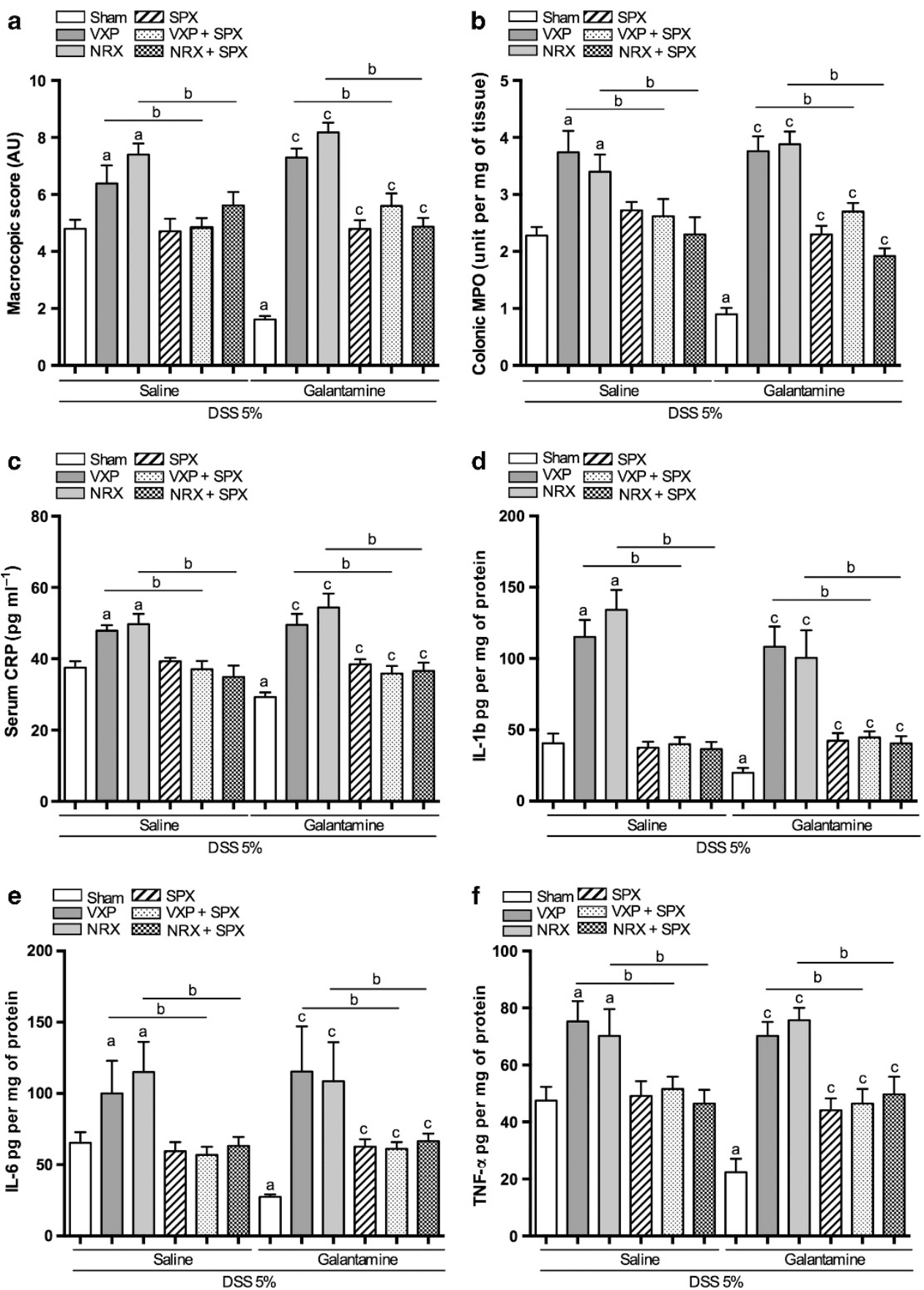

Figure 5 Galantamine's effects in mice with dextran sulfate sodium (DSS)-induced colitis are mediated through vagus nerve and splenic nerve signaling to the spleen. Vagotomy (VXP) and/or splenectomy (SPX), splenic neurectomy (NRX), and/or splenectomy (SPX) were performed 10 days before initiating galantamine $\left(4 \mathrm{mg} \mathrm{kg}^{-1}\right.$ day $^{-1}$, intraperitoneal (IP)) treatment and/or colitis induction as described in Methods. Sham represents data obtained in sham SPX mice, because no significant differences were determined between this group and any other sham group of animals; (a) macroscopic score; (b) colonic myeloperoxidase (MPO) activity; (c) serum C-reactive protein (CRP); (d) colonic interleukin (IL)-1 $\beta$ amount; (e) colonic IL-6 amount; and (f) colonic tumor necrosis factor (TNF)- $\alpha$ amount. Values are shown as means \pm s.e.m. Samples were collected on day 5 post-DSS treatment; mice per group $\geqslant 8$. ${ }^{\mathrm{a}} P<0.05$ as compared with the sham-saline-DSS-treated group, ${ }^{\mathrm{b}} P<0.05$ as compared with the VXP-DSS-treated group or the NRX-DSS-treated group, ${ }^{\mathrm{c}} P<0.05$ as compared with the sham-GAL-DSS-treated group. AU, arbitrary units.

AChE inhibitor, GAL has properties of an allosteric-positive modulator of nicotine, including $\alpha 7 \mathrm{nAChR} .^{30}$ Therefore, we evaluated the possibility that the stimulation of $\alpha 7 \mathrm{AChR}$ on splenic $\mathrm{CD} 11 \mathrm{c}^{+}$cells by GAL might contribute to antiinflammatory effects. Splenic CD11c ${ }^{+}$cells isolated from noncolitic mice were activated with lipopolysaccharide and treated with GAL or vehicle ex vivo. We did not observe any significant effect of GAL $(100 \mu \mathrm{M})$ on IL-12p40, IL-1 $\beta$, and IL-6 levels $(440 \pm 23$ vs. $435 \pm 41,640 \pm 78$ vs. $712 \pm 65,910 \pm 54$ vs. $876 \pm 44 \mathrm{pg} \mathrm{ml}^{-1}$, respectively) in these settings.

To further assess the effects of in vivo treatments and surgeries, we next examined the surface expression of MHC II 


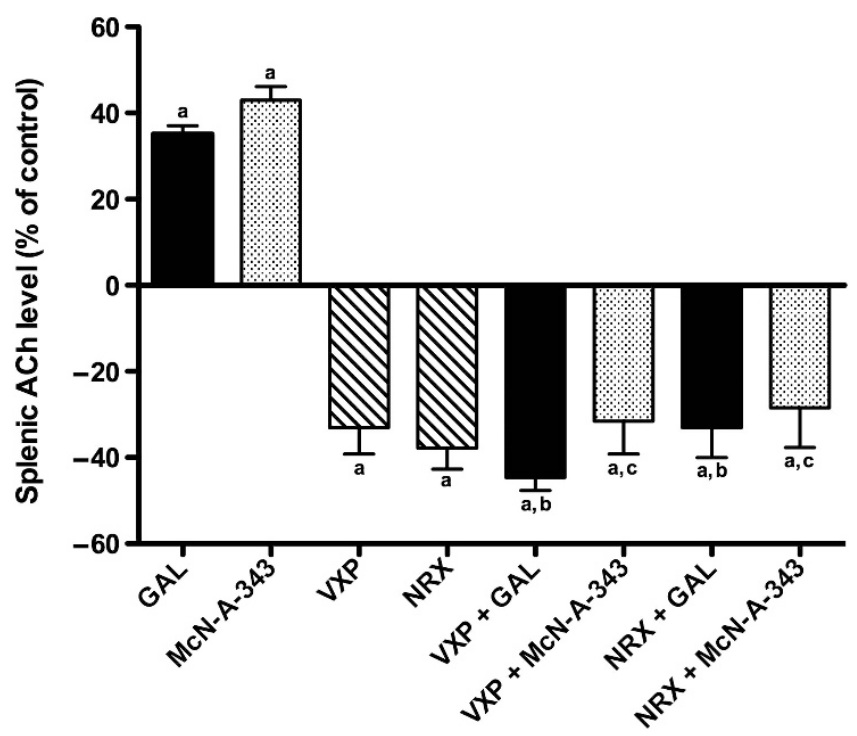

Figure 6 Cholinergic treatments with galantamine (GAL) or McN-A-343 result in increased splenic acetylcholine (ACh) levels, mediated through vagus nerve and splenic nerve signaling. Vagotomy (VXP) and/or splenic neurectomy (NRX) were performed 10 days before GAL ( $4 \mathrm{mg} \mathrm{kg}^{-1}$ day $^{-1}$, intraperitoneal (IP) for 6 days) or McN-A-343 ( $5 \mathrm{ng} \mathrm{kg}^{-1}$ day $^{-1}$, intracerebroventricular (ICV), for 6 days) treatments and splenic ACh levels were analyzed as described in Methods. Splenic ACh levels were determined on day 5 post-colitis induction with dextran sulfate sodium (DSS). In colitic control condition, the level of ACh was $1845 \pm 85 \mu \mathrm{m}$ per spleen. Values are shown as means \pm s.e.m., three independent experiments with four mice per group. ${ }^{a} P<0.05$ as compared with the control sham-DSS-treated group,

${ }^{\mathrm{b}} P<0.05$ as compared with the sham GAL-DSS-treated group, ${ }^{\mathrm{c}} P<0.05$ as compared with the sham MCN-A-343-DSS-treated group.

and costimulatory molecules (CD40, CD86, CD80). In the absence of colitis, naive splenic $\mathrm{CD} 11 \mathrm{c}^{+}$cells expressed low levels of CD40, CD86, CD80, and moderate levels of MHC II at cell surface. Upon activation of colonic inflammation, the surface expression of MHC II was upregulated (Figure 8a), and such upregulation was further augmented by VXP and NRX. CD40, CD80, and CD86 expressions were not modified (Figure 8b-d). GAL treatment decreased the level of MHC II in the splenic CD11 $\mathrm{c}^{+}$cells isolated from mice with colitis, and no beneficial effect of GAL treatment was found in the absence of an intact vagus or splenic nerve (Figure 8a). In the absence of colitis, no significant modification of the expression of the different markers was determined in the different groups (data not shown).

\section{DISCUSSION}

The results of this study clearly indicate that central cholinergic activation by AChE inhibitors or selective $\mathrm{mAChR}$ ligands results in a decreased susceptibility to experimental colitis. These protective effects are dependent on vagus nerve and splenic nerve signaling, which is mediated at a cellular level through cholinergic suppression of splenic DC activation.

In line with previous findings indicating a role for brain $\mathrm{mAChRs}$ in the central activation of the vagus nerve-mediated cholinergic anti-inflammatory pathway, ${ }^{20}$ exogenous GAL failed to reduce the severity of colitis in mice with pharmacological blockage (by AS) of central mAChRs. The lack of effects of AS or AMN alone on colitis severity suggests that the basal levels of ACh are not associated with protective effects through mAChR-mediated mechanisms. Furthermore, the therapeutic efficacy of McN-A-343 and MTT highlight exploiting central cholinergic activation by $\mathrm{M} 1 \mathrm{mAChR}$ agonists or M2mAChR antagonists as novel approaches to alleviate colitis. Although it is known that peripheral cholinergic activation may have some effect on colitis, ${ }^{31}$ in our study we found no evidence for a significant role of peripheral $\mathrm{mAChRs}$ in mediating protective cholinergic effects in the context of this experimental plan. Our findings that the effects of GAL and McN-A-343 are mediated through activation of the vagus nerve-based cholinergic anti-inflammatory pathway are consistent with experimental evidence pointing to a protective role of the vagus nerve against acute colitis in animal models. ${ }^{4,32,33}$ The disease-alleviating and anti-inflammatory effects of both GAL and McN-A-343 treatments were abrogated in mice with VXP, NRX, or mice with splenectomy. These results are in line with data demonstrating that vagus nerve stimulation fails to protect against septic shock in rats subjected to common celiac branch VXP, splenectomy, or NRX. ${ }^{25,34}$ Both therapeutics (GAL and McN-A-343) increased splenic ACh levels in mice and this effect was not observed in mice with VXP and NRX, indicating that the protective cholinergic effect is dependent on an interaction between the vagus nerve and the spleen. These results are consistent with recent observations that splenic ACh is released after vagus nerve activation, which can result in a paracrine effect on antigen-presenting cells and antiinflammatory effect during endotoxemia. ${ }^{25}$ Thus, our results identify a vagus nerve-to-spleen axis as an important mediator of the central cholinergic regulation of colitis severity. The importance of our findings was additionally substantiated by the similar profile of results in a DNBS-model, which broadens the therapeutic implications of cholinergic modalities in the regulation of intestinal inflammation.

Although previous observations clearly implicate peritoneal macrophages ${ }^{4,35}$ and splenic $\mathrm{T}$ cells ${ }^{36}$ in the context of the cholinergic anti-inflammatory pathway, it was not previously known whether changes in splenic $\mathrm{CD} 11 \mathrm{c}^{+}$cell responses have a role in regulating gut inflammation by cholinergic signaling. Our data demonstrate that activated vagus nerve signaling affects splenic $\mathrm{CD}_{11 \mathrm{c}^{+}}$cells' cytokine production and expression of MHC II, which subsequently may have an important role in the regulation of gut inflammation through the $\mathrm{CD} 4^{+}$T-cell population. This observation substantiates previous studies related to the importance of DCs in the context of experimental colitis ${ }^{37}$ and demonstrates amelioration of DSS-induced colitis by depletion of DCs after administration of diphtheria toxin in CD11c-DT receptor transgenic mice. ${ }^{17}$ This is in agreement with clinical data demonstrating that IBD is characterized by increased expression of IL-12p 40 , IL- $1 \beta$, and IL-6 pro-inflammatory cytokines in the gut and serum. ${ }^{38}$

Our results indicate that splenectomy does not affect colitis. This observation is in line with the hypothesis that appendicular lymphoid tissue, but not the spleen, contributes to the 

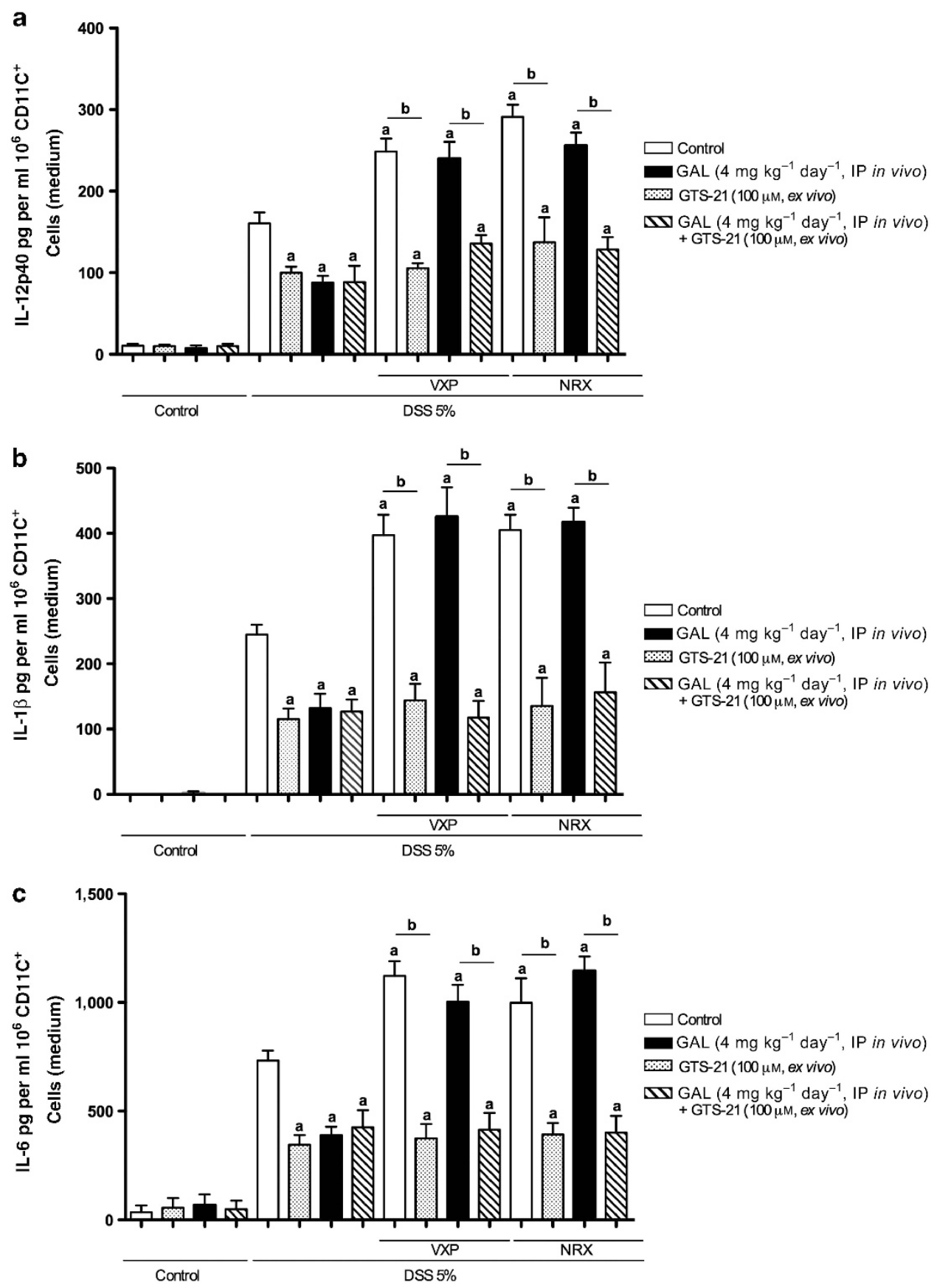

Figure 7 Effects of cholinergic treatment on splenic CD11c ${ }^{+}$cells' cytokine production in the context of dextran sulfate sodium (DSS)-induced colitis. (a) Interleukin (IL)-12p40, IL-1 $\beta$, and IL-6 production from dendritic cells. Splenic CD11c ${ }^{+}$cells were isolated from galantamine (GAL, $4 \mathrm{mg} \mathrm{kg}^{-1}$ day $^{-1}$, intraperitoneal (IP) for 6 days)-treated groups of colitic mice subjected to sham operation, vagotomy (VXP), or splenic neurectomy (NRX) on day 5 post-colitis induction. Splenic $\mathrm{CD}_{11 \mathrm{c}^{+}}$cells were also isolated from the groups of colitic mice subjected to sham operation, vagotomy (VXP), or splenic neurectomy (NRX) and incubated ex vivo with GTS-21 (a specific $\alpha 7 \mathrm{nAChR}$ agonist, $100 \mu \mathrm{m}$ ). IL-12p40, IL-1 $\beta$, and IL-6 were measured in media at $24 \mathrm{~h}$ following treatments. Values are shown as means \pm s.e.m., three independent experiments with four mice per group. ${ }^{a} P<0.05$ as compared with the DSS control group, ${ }^{b} P<0.05, n=8$.

development of colitis. ${ }^{39}$ It also suggests that while the gut is the site of disease initiation, the regulation of the cytokine release can be mediated via the spleen. As the vagus nerve has a tonic inhibitory activity on the release of pro-inflammatory cytokines such as IL- $1 \beta$ and IL- 6 , from the splenic DCs, its surgical interruption enhances the release of these cytokines from the spleen. This consequently results in exacerbation of the disease initiated within the colon and is abolished by VXP, NRX, or SPX. These findings are in line with the concept of neural signal modulation of mobile immune cell activation in the spleen. ${ }^{40}$ Although transiting the spleen, immune cells pass through its vascular matrix in proximity to neuronal endings arising from the splenic nerve. Thus, the inflammatory phenotype of immune cells might undergo neural modulation so that, when 

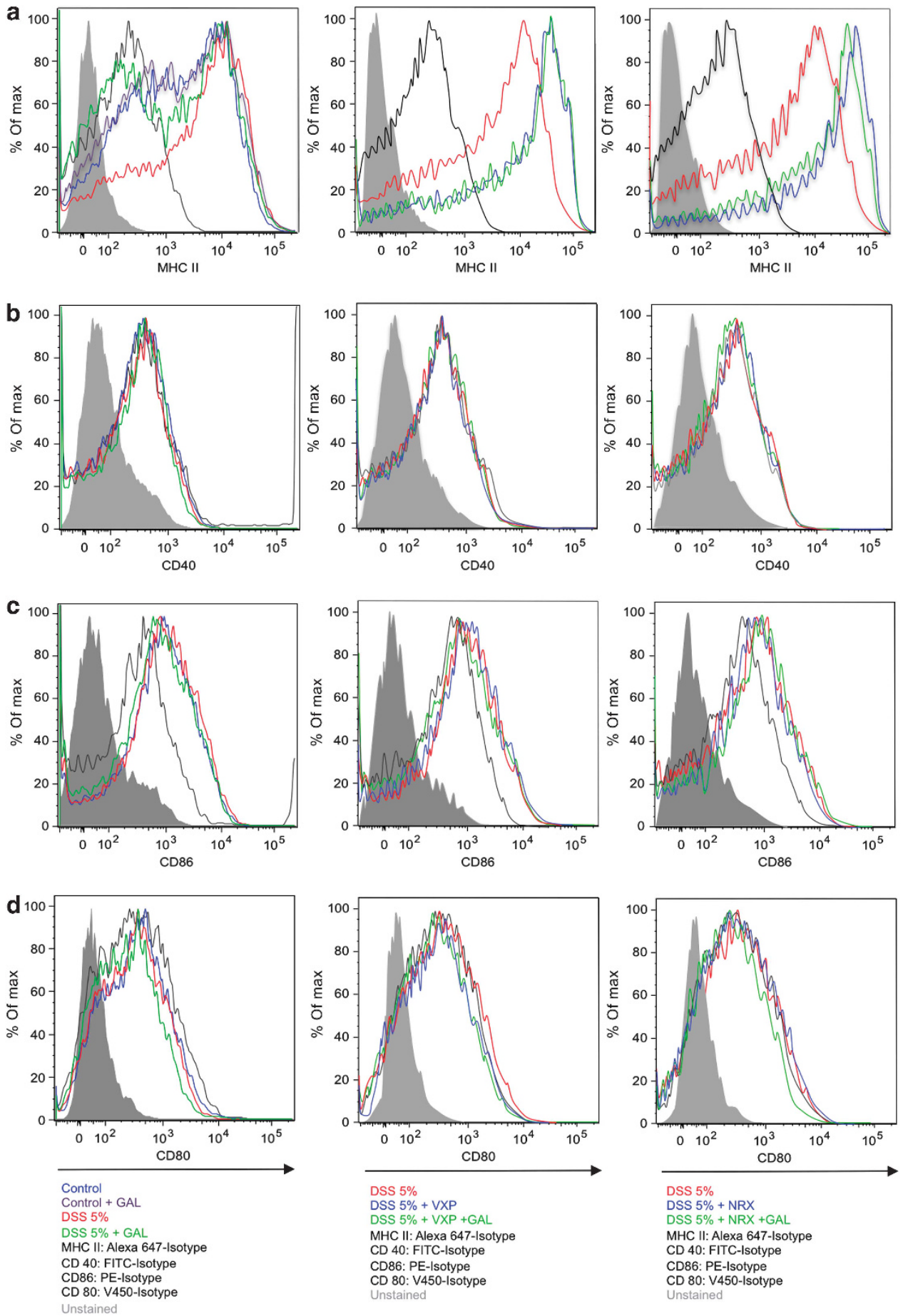

DSS $5 \%$
DSS $5 \%+V X P$

DSS $5 \%$ + VXP +GAL

MHC II: Alexa 647-Isotype

CD 40: FITC-Isotype

CD86: PE-Isotype

CD 80: V450-Isotype

Unstained

DSS $5 \%$

DSS $5 \%+$ NRX

DSS $5 \%+$ NRX + GAL

MHC II: Alexa 647-Isotype

CD 40: FITC-Isotype

CD86: PE-Isotype

CD 80: V450-Isotype

Unstained

Figure 8 Effect of cholinergic treatments with galantamine (GAL), vagotomy, and neurectomy on splenic CD11c ${ }^{+}$cells phenotype in the context of dextran sulfate sodium (DSS)-induced colitis. Using CD11 ${ }^{+}$MACS (magnetic-activated cell sorter)-positive selection, splenic CD11c ${ }^{+}$ cells were isolated from galantamine (GAL, $4 \mathrm{mg} \mathrm{kg}^{-1}$ day $^{-1}$, intraperitoneal (IP) for 6 days)-treated or vehicle groups of colitic mice subjected to sham operation, vagotomy (VXP), or splenic neurectomy (NRX) on day 5 post-colitis induction. Splenic CD11c ${ }^{+}$cell phenotype as characterized by median fluorescence intensity (percentage of max). (a) Major histocompatibility complex (MHC) II (Alexa-647), (b) CD40 (fluorescein isothiocyanate (FITC)), (c) CD80 (phycoerythrin (PE)), and (d) CD86 (V450) surface expression. Representative results from $n=4$ per group are shown.

exiting the spleen, these cells either fail to be recruited to zones of inflammation or fail to mediate damage. ${ }^{41}$ This concept has implications for understanding disease pathogenesis and the role of neural regulation in inflammatory conditions initiated or mediated by the trafficking and accumulation of inflammatory cells in peripheral tissues. Although it is theoretically possible that mesenteric lymphoid or lamina propria DCs may also be involved in mediating cholinergic effects in the context of colitis, our findings clearly point to a role of splenic $\mathrm{CD} 11 \mathrm{c}^{+}$cells in this regulation. However, the 
possible contribution of other circulating immune cell (i.e., granulocytes) might also be considered. This regulation could be dysfunctional in the context of IBD with autonomic impairment. Therefore, stimulating vagus nerve activity may contribute to restoring the anti-inflammatory neuroimmune regulation at the level of the spleen.

We have further highlighted the mediating role of the $\alpha 7 \mathrm{AChR}$ on splenic $\mathrm{CD}_{11 \mathrm{c}^{+}}$cells; a specific $\alpha 7 \mathrm{AChR}$ agonist downregulated ex vivo IL-12p40, IL- 6 , and TNF- $\alpha$ release. This corroborates data demonstrating that in vitro immature spleen DCs that mature in a nicotinic environment manifest lower endocytic and phagocytic activities. ${ }^{42}$ In vitro mature spleen DCs that are exposed to nicotine produce decreased levels of IL-12p40 and displayed reduced ability to induce T-Cell responses. ${ }^{43}$ Moreover, our data do not support the possibility that GAL may act as an allosteric modulator of the $\alpha 7 \mathrm{AChR}$ on splenic $\mathrm{CD} 11 \mathrm{c}^{+}$cells to directly affect their activation.

It should be acknowledged that apart from the spleen the vagus nerve targets the proximal colon. Therefore, it is theoretically possible that alterations in the inflammatory state in the vagus nerve-innervated proximal colon may affect the enteric nervous system activity in the remainder of the colon, ${ }^{44}$ which in turn may result in alterations in neurotransmitter release and suppression of inflammation. This may also account for the effect of vagus nerve signaling on colitis observed in this and other studies. ${ }^{4,32}$ However, in our study we did not observe significant effects of GAL or McN-A-343 in the absence of the spleen. It is also conceivable that other factors may contribute to the beneficial effects of these cholinergic treatments on colitis. A dominant sympathetic drive, which can simultaneously occur with impaired vagus nerve activity, has been associated with enhanced colonic inflammation. ${ }^{45}$ In addition, vagotomy alters lymphocyte trafficking ${ }^{46}$ and the number of mast cells in the gut $^{47}$ and influences gut physiology. ${ }^{48}$ Therefore, alteration of these parameters may also contribute to changes in colitic severity as a result of cholinergic, vagus nerve-mediated stimulation. Furthermore, the fact that we did not observe significant effects of GAL or McN-A-343 on IL-10 levels suggests that alterations in antiinflammatory cytokine levels do not have a role in mediating the beneficial effects of cholinergic modalities on colitis severity. This confirms previous data indicating no role of IL-10 in mediating cholinergic regulation of immune responses in models of endotoxemia and colitis. ${ }^{35,49}$ In addition, cholinergic treatments did not alter the stool consistency in control mice, indirectly pointing to a lack of significant effect on gut physiology.

Our findings prompt close consideration of the relationship between brain cholinergic activation and disease activity in patients with colonic inflammation and have important clinical relevance. Patients with colonic inflammatory conditions might be selected for novel treatment strategies, including: (1) centrally acting AChE inhibitors, which are already clinically approved for the treatment of Alzheimer's disease; (2) highly selective muscarinic receptor agonists or antagonists, ${ }^{6}$ which are being actively developed for the treatment of Alzheimer's disease and other neurodegenerative disorders; and (3) electrical vagus nerve stimulation, which is now being used for the treatment of refractory depression and epilepsy. ${ }^{50}$ The therapeutic potential of alternative techniques targeting an increase of efferent vagus nerve outflow, such as acupuncture $^{51,52}$ and zen, ${ }^{53,54}$ may also be studied in the context of colonic inflammation.

\section{METHODS}

Animals. Male C57BL/6 (7-9-weeks old) were purchased from Charles Rivers (Saint-Constant, QC, Canada) and maintained in the animal care facility at the University of Manitoba under specific pathogen-free conditions. No differences in food intake or body weight were observed between the groups. All experiments were approved by the University of Manitoba animal ethics committee (10-073) and conducted under the Canadian guidelines for animal research.

Induction of DSS and DNBS colitis. DSS (molecular weight, 40 kilodaltons: ICN Biomedicals, Burlington, ON, Canada) was added to the drinking water in a final concentration of $5 \%$ (wt/vol) for 5 days. ${ }^{5,56}$ Controls were all time-matched and consisted of mice that received normal drinking water only. Mean DSS consumption was noted per cage each day. For the DNBS study, mice were anesthetized with Isoflurane (Abbott, Abbott Park, IL). A 10-cm long PE-90 tubing (ClayAdam, Parsippany, NJ), attached to a tuberculin syringe, was inserted $3.5 \mathrm{~cm}$ into the colon. Colitis was induced by administration of $100 \mu \mathrm{l}$ of $4 \mathrm{mg}$ of DNBS solution (ICN Biomedicals) in 50\% ethanol and left for 3 days. ${ }^{49}$ Control mice (without colitis) received saline administration. Mice with colitis were supplied with $6 \%$ sucrose in drinking water to prevent dehydration.

Surgical procedures and drug treatments. Mice were anesthetized using ketamine (150 $\mathrm{mg} \mathrm{kg}^{-1}$, IP) and xylazine (10 $\mathrm{mg} \mathrm{kg}^{-1}$, IP). ICV implantation of the cannula, splenectomy (SPX), splenic neurectomy (NRX), or subdiaphragmatic bilateral vagotomy (VXP) was performed on the same day. ${ }^{4}$ In the sham-operated group: (1) mice implanted with the cannula received vehicle; (2) mice were anesthetized and laparotomy performed but the spleen was not removed; (3) splenic nerve was exposed but not cut; and (4) vagal trunks were exposed but not cut; however, a pyloroplasty was performed. The completeness of vagotomy was verified during post-mortem inspection of vagal nerve endings using microscopic inspection associated with a Bielschowsky silver staining. ${ }^{57}$ The completeness of neurectomy was verified post mortem by noradrenaline enzyme-linked immunosorbent assay in sham-operated and NRX animals. Mice were allowed to recover for 10 days. One day before initiation of colitis, pharmacological treatments started: GAL (1-4 $\mathrm{mg} \mathrm{kg}^{-1}$ day $^{-1}$, IP); Hup A: $\left(0.4 \mathrm{mg} \mathrm{kg}^{-1}\right.$ day $^{-1}$, IP, Sigma, Oakville, ON); AMN (injected $20 \mathrm{~min}$ before GAL, $4 \mathrm{mg} \mathrm{kg}^{-1} \mathrm{day}^{-1}$, IP, Sigma); and AS (injected $20 \mathrm{~min}$ before GAL, $4 \mathrm{mg} \mathrm{kg}^{-1} \mathrm{day}^{-1}$, IP Sigma). Micro-osmotic pumps (Alzet, Cupertino, CA) were filled with vehicle (saline $0.2 \%$ ), M1 mAChR agonist McN-A-343 (Sigma), or M2 mAChR antagonist MTT $\left(5 \mathrm{ng} \mathrm{day}^{-1}\right.$, Sigma) solution and placed as previously described. ${ }^{49}$

Characterization of inflammation. Disease activity index, macroscopic scores, and colonic damage were determined using a previously described scoring system for DSS colitis ${ }^{4,58}$ and for DNBS ${ }^{4,59}$ over 5 and 3 days, respectively. Samples were collected 5 or 3 days post activation associated with DSS or DNBS, respectively , and blood was collected by intracardiac puncture under isoflurane anesthesia. Formalin-fixed colon segments coming from the splenic flexure were stained with hematoxylin-eosin. ${ }^{4}$ Colonic MPO activity was determined following an established protocol. ${ }^{60}$ Serum C-reactive protein and colonic cytokine levels were determined using ELISA commercial kit (R\&D Systems, Minneapolis, MN). 
Acetylcholine detection. Five or 3 days post activation associated with DSS or DNBS, respectively, the amount of acetylcholine was measured using the acetylcholine assay kit (Amplex Red; Molecular Probes, Burlington, ON, Canada). This kit measures the amount of hydrogen peroxide (which in the presence of horseradish peroxidase leads to the oxidation of Amplex Red) produced through the oxidation of choline. The concentrations of choline and acetylcholine were determined using the software provided by the manufacturer (KC4; Bio-Tek, Winooski, WT).

Isolation of splenic $\mathrm{CD}_{11 c^{+}}$cells and culture. Five or 3 days post activation associated with DSS or DNBS, respectively, the spleens were digested in $2 \mathrm{mg} \mathrm{ml}^{-1}$ collagenase D (Roche Diagnostics, Meylan, France) in RPMI 1640 for $30 \mathrm{~min}$ at $37^{\circ} \mathrm{C}$. EDTA at $5 \mathrm{~mm}$ was added during the last $5 \mathrm{~min}$ to disrupt $\mathrm{DC}-\mathrm{T}$ cell complexes, and the cell suspension was filtered. Total splenocytes after red blood cell lysis with ACK lysis buffer (150 mm NH4Cl, $10 \mathrm{~mm}$ KHCO3, $0.1 \mathrm{~mm}$ EDTA) were incubated with $\mathrm{CD} 11 \mathrm{c}^{+}$microbeads (Miltenyi Biotec, Auburn, CA) for $15 \mathrm{~min}$ at $48^{\circ} \mathrm{C}$. The cells were then washed, resuspended in cell separation buffer (Dulbecco's phosphate-buffered saline without $\mathrm{Ca} 21$ and Mg 21 containing 2\% fetal bovine serum and 2 mM EDTA, Life Technology, Burlington, ON, Canada), and passed through magnetic columns for positive selection. After passing consecutively through two columns, the collected splenic $\mathrm{CD} 11 \mathrm{c}^{+}$cell preparations showed $>95 \%$ purity. Splenic CD11 ${ }^{+}$cell isolated from the different groups of mice were cultured in complete RPMI 1640 medium containing $10 \%$ heat-inactivated fetal bovine serum, $25 \mathrm{mg} \mathrm{ml}^{-1}$ gentamicin, $2 \mathrm{~mm}$ L-glutamine in 12 -well plates at $1 \times 10^{6}$ cells per well for $24 \mathrm{~h}$, and the supernatants were measured for IL-12p40, IL-6, and TNF- $\alpha$ by ELISA (R\&D Systems).

In some experiments, lipopolysaccharide (Sigma) was added to the cultures at a final concentration of $100 \mathrm{ng} \mathrm{ml}^{-1}$. In a separated set, GAL or the a7nAChR agonist GST-21 was added to the medium at a final concentration of $10^{-6} \mathrm{M}$.

Flow cytometry. Surface staining of MACS-isolated splenic CD11c ${ }^{+}$ cell (MHC II-Alexa 647, CD40-FITC, CD86-PE, CD80-V450) (BD Biosciences, Mississauga, $\mathrm{ON}$, Canada) of different in vivo treatments were subjected to standard multi-color flow cytometry procedures. ${ }^{61}$ In brief, fluorescent-labeled antibodies were added to the splenic $\mathrm{CD} 11 \mathrm{c}^{+}$cell $\left(10^{6}\right)$ and incubated at $4{ }^{\circ} \mathrm{C}$ for $30 \mathrm{~min}$ in all surface staining procedures. After excessive washing in flow buffer to remove unbound antibodies, the cells were acquired in a BD FACS Calibur Flow Cytometer. Cell viability was assessed using DAPI (4,6-diamidino-2-phenylindole). Data analysis were performed using the Flowjo software (TreeStar, Ashland, OR).

Statistical analysis. Results are presented as means \pm s.e.m. Statistical analysis was performed using one- or two-way analysis of variance followed by the Tukey-Kramer multiple comparison post hoc analysis and a $P$-value of $<0.05$ considered significant with $n=8-12$ depending on the groups tested (Prism 4, GraphPad, La Jolla, CA).

SUPPLEMENTARY MATERIAL is linked to the online version of the paper at http://www.nature.com/mi

\section{ACKNOWLEDGEMENTS}

We thank Dr Charles N. Bernstein and Dr Stephen M. Collins for their discussion and continuous support. The work was supported by the Manitoba Health Research Council, the University of Manitoba, the Canada Foundation for Innovation, and the National Institute of General Medical Sciences, National Institutes of Health (1RO1GM089807 to K.J.T.).

\section{AUTHOR CONTRIBUTION}

JE.G. designed the study. H.J., M.F.R., and JE.G., carried out the majority of the experiments; B.L. carried out the remaining experiments. K.J.T., V.A.P., and JE.G. analyzed the data. JE.G. wrote the first draft of the manuscript. K.J.T. and V.A.P. reviewed the draft and provided comments. All authors approved the final submission.

\section{DISCLOSURE}

The authors declared no conflict of interest.

c 2014 Society for Mucosal Immunology

\section{REFERENCES}

1. Molodecky, N.A. et al. Increasing incidence and prevalence of the inflammatory bowel diseases with time, based on systematic review. Gastroenterology 142, 46-54. e42; quiz e30 (2012).

2. Strober, W. \& Fuss, I.J. Proinflammatory cytokines in the pathogenesis of inflammatory bowel diseases. Gastroenterology 140, 1756-1767 (2011).

3. Perrier, C. \& Rutgeerts, P. Cytokine blockade in inflammatory bowel diseases. Immunotherapy 3, 1341-1352 (2011).

4. Ghia, J.E., Blennerhassett, P., Kumar-Ondiveeran, H., Verdu, E.F. \& Collins, S.M. The vagus nerve: a tonic inhibitory influence associated with inflammatory bowel disease in a murine model. Gastroenterology 131, 1122-1130 (2006).

5. Goldstein, R.S. et al. Cholinergic anti-inflammatory pathway activity and High Mobility Group Box-1 (HMGB1) serum levels in patients with rheumatoid arthritis. Mol. Med. 13, 210-215 (2007).

6. The, F. et al. Central activation of the cholinergic anti-inflammatory pathway reduces surgical inflammation in experimental post-operative ileus. $\mathrm{Br}$. J. Pharmacol. 163, 1007-1016 (2011).

7. Borovikova, L.V. et al. Role of vagus nerve signaling in CNI-1493-mediated suppression of acute inflammation. Auton. Neurosci. 85, 141-147 (2000).

8. Van Der Zanden, E.P., Boeckxstaens, G.E. \& de Jonge, W.J. The vagus nerve as a modulator of intestinal inflammation. Neurogastroenterol. Motil. 21, 6-17 (2009).

9. Pavlov, V.A. \& Tracey, K.J. The vagus nerve and the inflammatory reflexlinking immunity and metabolism. Nat. Rev. Endocrinol. 8, 743-754 (2012).

10. Lindgren, S., Stewenius, J., Sjolund, K., Lilja, B. \& Sundkvist, G. Autonomic vagal nerve dysfunction in patients with ulcerative colitis. Scand. J. Gastroenterol. 28, 638-642 (1993).

11. Meregnani, J. et al. Anti-inflammatory effect of vagus nerve stimulation in a rat model of inflammatory bowel disease. Auton. Neurosci. 160, 82-89 (2011).

12. Cosnes, J. Tobacco and IBD: relevance in the understanding of disease mechanisms and clinical practice. Best Pract. Res. Clin. Gastroenterol. 18, 481-496 (2004).

13. Frolkis, A. et al. Environment and the inflammatory bowel diseases. Can. J. Gastroenterol. 27, e18-e24 (2013).

14. Drakes, M.L., Blanchard, T.G. \& Czinn, S.J. Colon lamina propria dendritic cells induce a proinflammatory cytokine response in lamina propria T cells in the SCID mouse model of colitis. J. Leukoc. Biol. 78, 1291-1300 (2005).

15. Ikeda, Y., Ueno, A., Naraba, H. \& Oh-ishi, S. Involvement of vanilloid receptor VR1 and prostanoids in the acid-induced writhing responses of mice. Life Sci. 69, 2911-2919 (2001).

16. te Velde, A.A. et al. Increased expression of DC-SIGN + IL-12+IL-18+ and CD83 + IL-12-IL-18- dendritic cell populations in the colonic mucosa of patients with Crohn's disease. Eur. J. Immunol. 33, 143-151 (2003).

17. Berndt, B.E., Zhang, M., Chen, G.H., Huffnagle, G.B. \& Kao, J.Y. The role of dendritic cells in the development of acute dextran sulfate sodium colitis. J. Immunol. 179, 6255-6262 (2007).

18. Yoshikawa, $\mathrm{H}$. et al. Nicotine inhibits the production of proinflammatory mediators in human monocytes by suppression of I-kappaB phosphorylation and nuclear factor-kappaB transcriptional activity through nicotinic acetylcholine receptor alpha7. Clin. Exp. Immunol. 146, 116-123 (2006).

19. Kawashima, K., Yoshikawa, K., Fujii, Y.X., Moriwaki, Y. \& Misawa, H. Expression and function of genes encoding cholinergic components in murine immune cells. Life Sci. 80, 2314-2319 (2007)

20. Pavlov, V.A. et al. Central muscarinic cholinergic regulation of the systemic inflammatory response during endotoxemia. Proc. Natl. Acad. Sci. USA 103, 5219-5223 (2006).

21. Pavlov, V.A. et al. Brain acetylcholinesterase activity controls systemic cytokine levels through the cholinergic anti-inflammatory pathway. Brain Behav. Immun. 23, 41-45 (2009).

22. Ellis, J.M. Cholinesterase inhibitors in the treatment of dementia. J. Am. Osteopath. Assoc. 105, 145-158 (2005). 
23. Waldburger, J.M. et al. Spinal p38 MAP kinase regulates peripheral cholinergic outflow. Arthritis Rheum. 58, 2919-2921 (2008).

24. Shifrin, H., Nadler-Milbauer, M., Shoham, S. \& Weinstock, M. Rivastigmine alleviates experimentally induced colitis in mice and rats by acting at central and peripheral sites to modulate immune responses. PLoS One 8, e57668 (2013).

25. Rosas-Ballina, M. et al. Acetylcholine-synthesizing $T$ cells relay neural signals in a vagus nerve circuit. Science 334, 98-101 (2011).

26. Bickel, U., Thomsen, T., Fischer, J.P., Weber, W. \& Kewitz, H. Galanthamine: pharmacokinetics, tissue distribution and cholinesterase inhibition in brain of mice. Neuropharmacology 30, 447-454 (1991).

27. Stillman, M.J., Shukitt-Hale, B., Kong, R.M., Levy, A. \& Lieberman, H.R. Elevation of hippocampal extracellular acetylcholine levels by methoctramine. Brain Res. Bull. 32, 385-389 (1993).

28. Lachowicz, J.E. et al. Facilitation of acetylcholine release and improvement in cognition by a selective M2 muscarinic antagonist, SCH 72788. Life Sci. 68, 2585-2592 (2001).

29. Ghia, J.E., Blennerhassett, P. \& Collins, S.M. Vagus nerve integrity and experimental colitis. Am. J. Physiol. Gastrointest. Liver Physiol. 293, G560-G567 (2007).

30. Texido, L. et al. Effect of galantamine on the human alpha7 neuronal nicotinic acetylcholine receptor, the Torpedo nicotinic acetylcholine receptor and spontaneous cholinergic synaptic activity. Br. J. Pharmacol. 145, 672-678 (2005).

31. Miceli, P.C. \& Jacobson, K. Cholinergic pathways modulate experimental dinitrobenzene sulfonic acid colitis in rats. Auton. Neurosci. 105, 16-24 (2003).

32. Mazelin, L., Theodorou, V., More, J., Fioramonti, J. \& Bueno, L. Protective role of vagal afferents in experimentally-induced colitis in rats. J. Auton. Nerv. Syst. 73, 38-45 (1998).

33. van Westerloo, D.J. et al. The vagus nerve and nicotinic receptors modulate experimental pancreatitis severity in mice. Gastroenterology 130, 1822-1830 (2006).

34. Xue, N., Liang, H., Yao, H., Song, X.M. \& Li, J.G. The role of spleen in vagus nerve stimulation for treatment against septic shock in rats. Zhongguo Wei Zhong Bing Ji Jiu Yi Xue 23, 263-266 (2011).

35. Borovikova, L.V. et al. Vagus nerve stimulation attenuates the systemic inflammatory response to endotoxin. Nature 405, 458-462 (2000).

36. Karimi, K., Bienenstock, J., Wang, L. \& Forsythe, P. The vagus nerve modulates CD4 + Tcell activity. Brain Behav. Immun. 24, 316-323 (2010).

37. Abe, K. et al. Conventional dendritic cells regulate the outcome of colonic inflammation independently of T cells. Proc. Natl. Acad. Sci. USA 104, 17022-17027 (2007).

38. Sanchez-Munoz, F., Dominguez-Lopez, A. \& Yamamoto-Furusho, J.K. Role of cytokines in inflammatory bowel disease. World J. Gastroenterol. 14, 4280-4288 (2008).

39. Krieglstein, C.F. et al. Role of appendix and spleen in experimental colitis. J. Surg. Res. 101, 166-175 (2001).

40. Tracey, K.J. Understanding immunity requires more than immunology. Nat. Immunol. 11, 561-564 (2010).

41. Saeed, R.W. et al. Cholinergic stimulation blocks endothelial cell activation and leukocyte recruitment during inflammation. J. Exp. Med. 201, 1113-1123 (2005).

42. Guinet, E., Yoshida, K. \& Nouri-Shirazi, M. Nicotinic environment affects the differentiation and functional maturation of monocytes derived dendritic cells (DCs). Immunol. Lett. 95, 45-55 (2004).

43. Nouri-Shirazi, M., Tinajero, R. \& Guinet, E. Nicotine alters the biological activities of developing mouse bone marrow-derived dendritic cells (DCs). Immunol. Lett. 109, 155-164 (2007).
44. Jacobson, K., McHugh, K. \& Collins, S.M. Experimental colitis alters myenteric nerve function at inflamed and noninflamed sites in the rat. Gastroenterology 109, 718-722 (1995).

45. McCafferty, D.M., Wallace, J.L. \& Sharkey, K.A. Effects of chemical sympathectomy and sensory nerve ablation on experimental colitis in the rat. Am. J. Physiol. 272, G272-G280 (1997).

46. Antonica, A., Ayroldi, E., Magni, F. \& Paolocci, N. Lymphocyte traffic changes induced by monolateral vagal denervation in mouse thymus and peripheral lymphoid organs. J. Neuroimmunol. 64, 115-122 (1996).

47. Gottwald, T.P., Lhotak, S. \& Stead, R.H. Effects of subdiaphragmatic vagotomy on mucosal mast cell densities in stomach and jejunum of rats. Adv. Exp. Med. Biol. 371A, 303-306 (1995).

48. Dapoigny, M., Cowles, V.E., Zhu, Y.R. \& Condon, R.E. Vagal influence on colonic motor activity in conscious nonhuman primates. Am. J. Physiol. 262, G231-G236 (1992).

49. Ghia, J.E., Blennerhassett, P. \& Collins, S.M. Impaired parasympathetic function increases susceptibility to inflammatory bowel disease in a mouse model of depression. J. Clin. Invest. 118, 2209-2218 (2008).

50. Diamond, A., Kenney, C. \& Jankovic, J. Effect of vagal nerve stimulation in a case of Tourette's syndrome and complex partial epilepsy. Mov. Disord. 21, 1273-1275 (2006).

51. Huang, S.T., Chen, G.Y., Lo, H.M., Lin, J.G., Lee, Y.S. \& Kuo, C.D. Increase in the vagal modulation by acupuncture at neiguan point in the healthy subjects. Am. J. Chin. Med. 33, 157-164 (2005).

52. Sakai, S., Hori, E., Umeno, K., Kitabayashi, N., Ono, T. \& Nishijo, H. Specific acupuncture sensation correlates with EEGs and autonomic changes in human subjects. Auton. Neurosci. 133, 158-169 (2007).

53. Kubota, Y. et al. Frontal midline theta rhythm is correlated with cardiac autonomic activities during the performance of an attention demanding meditation procedure. Brain Res. Cogn. Brain Res. 11, 281-287 (2001).

54. Paul-Labrador, $\mathrm{M}$ et al. Effects of a randomized controlled trial of transcendental meditation on components of the metabolic syndrome in subjects with coronary heart disease. Arch. Intern. Med. 166, 1218-1224 (2006).

55. Ghia, J.E., Galeazzi, F., Ford, D.C., Hogaboam, C.M., Vallance, B.A. \& Collins, S.M. Role of M-CSF dependent macrophages in colitis is driven by the nature of the inflammatory stimulus. Am. J. Physiol. Gastrointest. Liver Physiol. 294, 770-777 (2008).

56. Okayasu, I., Hatakeyama, S., Yamada, M., Ohkusa, T., Inagaki, Y. \& Nakaya, R. A novel method in the induction of reliable experimental acute and chronic ulcerative colitis in mice. Gastroenterology 98, 694-702 (1990).

57. Rosenwald, A., Reusche, E., Ogomori, K. \& Teichert, H.M. Comparison of silver stainings and immunohistology for the detection of neurofibrillary tangles and extracellular cerebral amyloid in paraffin sections. Acta Neuropathol. (Berl) 86, 182-186 (1993).

58. Cooper, H.S., Murthy, S.N., Shah, R.S. \& Sedergran, D.J. Clinicopathologic study of dextran sulfate sodium experimental murine colitis. Lab. Invest. 69, 238-249 (1993).

59. Appleyard, C.B. \& Wallace, J.L. Reactivation of hapten-induced colitis and its prevention by anti-inflammatory drugs. Am. J. Physiol. 269, G119-G125 (1995).

60. Boughton-Smith, N.K., Wallace, J.L. \& Whittle, B.J. Relationship between arachidonic acid metabolism, myeloperoxidase activity and leukocyte infiltration in a rat model of inflammatory bowel disease. Agents Actions 25 , 115-123 (1988).

61. Zhang, L, Procuik, M, Fang, T \& Kung, SK. Functional analysis of the quantitative expression of a costimulatory molecule on dendritic cells using lentiviral vector-mediated RNA interference. J. Immunol. Methods 344, 87-97 (2009). 